\title{
REVIEWER ACKNOWLEDGMENT
}

The Editors gratefully acknowledge the generous assistance of the following reviewers who served The American Journal of Pathology between January 1 and December 31, 2014.

\author{
Hanna E. Abboud \\ Clara Abraham \\ David Abraham \\ Dale R. Abrahamson \\ Hans Acha-Orbea \\ Marc Achen \\ Ted S. Acott \\ Devendra K. Agrawal \\ Pankaj B. Agrawal \\ Nihal Ahmad \\ Kurt H. Albertine \\ John F. Alcorn \\ Graeme J. Alexander \\ J. Steven Alexander \\ Holly M. Algood \\ Hydar Ali \\ Julio Aliberti \\ Malcolm Alison \\ Kari Alitalo \\ Emad S. Alnemri \\ Charles E. Alpers \\ Gianfranco Alpini \\ Peter F. Ambros \\ Hans-Joachim Anders \\ Robert E. Anderson \\ Thomas Andl \\ Sebastiano Andò \\ Simone Anfossi \\ Brian H. Annex \\ Daniel Anthony \\ David A. Antonetti \\ Andrew Aplin \\ Rajendra S. Apte \\ Udayan Apte \\ Robert M. Aris \\ Agnes Arthur \\ Atsushi Asakura \\ Mohammad Asghar \\ Aristotelis Astrinidis \\ Sergei P. Atamas \\ Hellmut G. Augustin \\ K. Frank Austen \\ Gustavo E. Ayala \\ Salman Azhar \\ Ulrik T. Baandrup \\ Hans J. Baelde \\ Andrew D. Baines
}

Egbert Bakker
Barbara J. Ballermann
Itamar Barash
Andreea Barbu
Philip M. Barger
David L. Bartlett
Armando Bartolazzi
David P. Basile
Heinz Baumann
Kayla J. Bayless
Haydee Bazan
Jaideep Behari
Fariba Behbod
Aaron W. Bell
Ariela Benigni
Robert Bergquist
Pascal Bernatchez
Francesco Bertoni
Cagri Besirli
Konrad Beyreuther
Vineet Bhandari
Sanjoy K. Bhattacharya
Vishakha Bhave
Jinsong Bian
Diane Bielenberg
Daniel Bikle
Markus Bitzer
Rolf Bjerkvig
Stephen M. Black
Alexander Bobik
Marie-Luce Bochaton-Piallat
Manfred Boehm
Odile Boespflug-Tanguy
Philippe Boeuf
Rudolf Bohm
Dean Bok
Vera Bonilha
Gloria Bonuccelli
W. Henry Boom
Maarten C. Bosland
Kevin E. Bove
Yuri Bozzi
Amy Bradshaw
Margaret Brandwein-Gensler
Derek P. Brazil
Donna Brennan-Crispi
Catherine A. Brissette

Egbert Bakker

Steven L. Brody

Neil Brody

J. David Brook

Patrick D. Brophy

Celia F. Brosnan

Charles Brown

Susan Brown

Robert Brown

Melissa A. Brown

Kyle E. Brown

H. Alex Brown

Corrie Brown

William Brunken

Herbert Budka

Stéphane Burtey

Louis Buscail

Wade A. Bushman

Julia Busik

Igor Butovich

Ralph Buttyan

Wim A. Buurman

Lu Cai

Ruth Caldwell

John W. Calvert

Matthew Campbell

Qi Cao

Michael S. Caplan

Mairead A. Carroll

Steven L. Carroll

Amelia Casamassimi

Doris Cassio

Carlos Caulin

Gediminas Cepinskas

Brahim Chaqour

Nicolas Charlet-Berguerand

Christiane Charriaut-Marlangue

Bénédicte Chazaud

Sylvain Chemtob

Jun Chen

Yu Chen

Yabing Chen

C. Yan Cheng

Indumathi Chennamsetty

Stephen W. Chensue

Tsutomu Chiba
Kathleen R. Cho

Augustine M.K. Choi

Louise T. Chow

Sanjib Chowdhury

Itay Chowers

Erik I. Christensen

Arthur C. Chung

Leland W. Chung

Giuliano Ciarimboli

Maria C. Cid

Richard A. Clark

Erika C. Claud

Robert J. Coffey, Jr.

Annie Colberg-Poley

William B. Coleman

Sean P. Colgan

Phillippe Connes

Denise C. Connolly

Kip Connor

Edward M. Conway

H. Terence Cook

Linda Cook

Bryan L. Copple

Leslie Costello

Richard J. Cote

Elizabeth Cothren

Richard Coward

Gregory A. Cox

Scott D. Cramer

Isabel J. Crane

James M. Crawford

Katalin Csiszar

Juan R. Cubillos-Ruiz

Edna Cukierman

Zoran Culig

Claus Cursiefen

Jeffrey L. Curtis

Christopher W. Cutler

Garry Cutting

Mark J. Czaja

Robert J. D'Amato

Jeanine D'Armiento

Thomas M. D'Hooghe

Mariana D. Dabeva

Zhiyu Dai

James B. Dale

Howard Dang 
Darlene Dartt

Kaustubh Datta

Ben Davidson

Nicholas O. Davidson

Jeffrey M. Davidson

Mark de Caestecker

Frank R. de Gruijl

Robb U. de Iongh

Daphne De Jong

Cintia S. de Paiva

J. Brian de Souza

Abbe N. de Vallejo

Francesco Del Galdo

Stefano Del Prato

Laurie D. DeLeve

Horace M. DeLisser

Constantinos Demopoulos

Sylvie H. Demouzon

Eric Y. Denkers

Timothy Denning

Elena Deryugina

Pallavi R. Devchand

Antonio Di Cristofano

Dolores Di Vizio

Dennis W. Dickson

Paul E. DiCorleto

Gabriel E. DiMattia

Wen-Xing Ding

Debra I. Diz

Vladislav Dolgachev

Paari Dominic

Horng-Yunn Dou

Duska Dragun

Ronny I. Drapkin

Sascha Drewlo

Ed Dudley

Stefan Duensing

Colin R. Dunstan

Karen Dwyer

Kathryn A. Eaton

Klaus T. Ebnet

Anne Eckert

Florian Eichler

Joseph El Khoury

Peter M. Elias

Karen L. Elkins

David M. Engman

Motomi Enomoto-Iwamoto

Mark L. Entman

Ozlem Er

Jan Jaap H. Erwich

Stephen M. Factor

Janet A. Fairley

DeLisa Fairweather

Domenick Falcone

Jianglin Fan

Giamila Fantuzzi

Harrison W. Farber

Asgerally T. Fazleabas

Kenneth R. Feingold

Diane Felsen

Gen-Sheng Feng

Jenifer I. Fenton

Stefan Fichtner-Feigl

Alan Fine

Mitchell P. Fink

Jacob N. Finkelstein
Gary S. Firestein

Steven K. Fisher

Melinda Fitzgerald

Howard B. Fleit

Erica L. Fletcher

Thomas J. Flotte

Rebecca D. Folkerth

Robert J. Fontana

Stuart J. Forbes

Kimberly E. Foreman

John V. Forrester

Mark E. Fortini

Cynthia Fourgeux

Nikolaos G. Frangogiannis

Philippe G. Frank

Michael A. Frohman

Yang-Xin Fu

Rudolf Fuchshofer

Robert S. Fujinami

Tohru Fukai

Seiji Fukumoto

Priscilla A. Furth

Masutaka Furue

Giulio Gabbiani

Elena V. Galkina

Vadivel Ganapathy

C. Shekhar R. Gandhi

Samuel Gandy

Ramesh K. Ganju

Allen C. Gao

Bin Gao

Zhijian Gao

Elizabeth E. Gardiner

Humphrey Gardner

Peter J. Gaskill

Fengxia Ge

Howard E. Gendelman

Spiro Getsios

Godfrey S. Getz

Robert H. Getzenberg

Andrew Gewirtz

Paramita Ghosh

Kalpana Ghoshal

Cecilia M. Giachelli

Roben G. Gieling

Danielle Gilkes

Gary S. Gilkeson

Michael Gilmore

Robert D. Gilmore

Pere Gines

Antonio Giordano

Ilene Gipson

Mahasweta Girgenrath

George Giudice

Tibor T. Glant

Glenda Gobe

Ralf Gold

Todd E. Golde

James E. Goldman

Mary B. Goldring

Xiaohua Gong

Frank J. Gonzalez

Leo I. Gordon

Gregory J. Gores

John D. Gottsch

Abdelilah S. Gounni

Gunnar K. Gouras
Joseph P. Grande

Sergei A. Grando

D. Neil Granger

Henk L. Granzier

Erin A. Greaves

David G. Greenhalgh

John Greenwood

Anna Greka

Greta Guarda

Christophe Guignabert

Larry J. Guilbert

Grace Guo

Sanjeev Gupta

Denis C. Guttridge

John R. Guyton

Bernard Haendler

Abraham A. Hakimi

Sandra Halonen

Carl V. Hamby

Anne Hamik

Steve Hann

Laura A. Hansen

Joshua M. Hare

David A. Harris

David C. Harris

Edward Harris

Arndt Hartmann

Kevan L. Hartshorn

Cristina Has

Jeffrey D. Hasday

Takanori Hattori

Lars F. Hedin

Richard G. Hegele

Travis W. Hein

Paul G. Hellewell

Gary M. Hellmann

Martin E. Hemler

Doris Hendig

Mary J.C. Hendrix

Michael Herfs

Meenhard Herlyn

Betsy Herold

Stephen M. Hewitt

Paul J. Higgins

Charles E. Hill

Mikko Hiltunen

Okio Hino

Vanessa M. Hirsch

Karen K. Hirschi

Jeffrey B. Hodgin

Kairbaan M. Hodivala-Dilke

Peter Hohenstein

Morley Hollenberg

Harry Holthofer

Paul Holvoet

Jonathon W. Homeister

Ann M. Hopkins

Akira Horii

Jeffrey C. Horowitz

Anton J. Horrevoets

Chantal Housset

Ralph H. Hruban

Andelko Hrzenjak

Hong-Yuan Huang

Tobias B. Huber

Jeremy Hughes

Christian Hugo
M. Louise Hull

Benjamin D. Humphreys

Berthold Huppertz

Andrea Huwiler

Philip M. Iannaccone

Steven Idell

John S. Ikonomidis

David H. Ingbar

Juan Iovanna

Renato V. Iozzo

John Isaacs

Akemi Ishida-Yamamoto

Yoshifumi Itoh

Michael M. Ittmann

Andrei I. Ivanov

Angelo A. Izzo

Nabila Jabrane-Ferrat

Chaim O. Jacob

Hartmut W. Jaeschke

Martine J. Jager

Christophe Jamin

Sabina Janciauskiene

Paul Janssen

Bernard Jasmin

Marie Jeansson

Jae-Wook Jeong

Carmen Jeronimo

James V. Jester

Sergio A. Jimenez

Christian Jobin

Achim Joerres

Peter Jones

Phil Jones

Adrian M. Jubb

Andreas Junker

Elliott Kagan

Taku Kambayashi

Motoi Kanagwa

Alpdogan Kantarci

Yashpal S. Kanwar

Winston W.-Y. Kao

William J. Karpus

S. Anath Karumanchi

Clifford E. Kashtan

Hiroaki Kataoka

Masaru Katoh

C. Dirk Keene

Dontscho Kerjaschki

Venkateshwar Keshamouni

Christopher G. Kevil

Bilion Khambu

Saleem A. Khan

Farrah Kheradmand

Konstantin Khrapko

Yujiro Kida

Laurie Kilpatrick

Jae-Sung Kim

Jayoung Kim

Mark S. Kindy

George L. King

Dimitra Kiritsi

Tatiana Kisseleva

Richard Kitching

Beatrice S. Knudsen

Erik S. Knudsen

Warren Knudson

Ruth Knuechel-Clarke 
Lester Kobzik

Stefan Koch

Olivier Kocher

Rohit Kohli

Martin R. Kolb

Joe N. Kornegay

Constantinos Koumenis

Michael H. Koval

Aleksander Krag

Michael Kremer

Wilhelm Kriz

Laurie T. Krug

Regina Kuliawat

A. Pratap Kumar

Ashok Kumar

Hiroki Kuniyasu

Calvin Kuo

Lih Kuo

Robert J. Kurman

Christian Kurts

Michael Kyba

Natasha Kyprianou

Veli-Matti Kähäri

Ezio Laconi

Nigel G. Laing

Lars Larsson

Hans Lassmann

Dennis Lau

Lester F. Lau

Robert M. Lavker

Abdel Lawendy

Elizabeth Lawlor

Pedro A. Lazo

Andrew Leask

Andrea C. LeBlanc

Franck Lebrin

Chun Geun Lee

Chung Lee

Huei Lee

William Leenders

Rudy Leibel

Samuel J. Leibovich

Cynthia A. Lemere

Wayne I. Lencer

Alex B. Lentsch

Klaus F. Ley

Lisette Leyton

De-Quan Li

Pawel P. Liberski

Andrew Lichtman

Graham Lieschke

Keith L. Ligon

Jan HN Lindeman

Volkhard Lindner

Rick Livesey

Alexander Ljubimov

Dan Longley

Joseph A. Lorenzo

Nancy A. Louis

Shelly C. Lu

Friedrich C. Luft

Nicholas W. Lukacs

Jian-Hua Luo

Gerard A. Lutty

James P. Luyendyk

Thomas Ma

Fabiana Machado
Fernando Macian

Wallace K. MacNaughton

Thomas M. Magin

Cynthia M. Magro

Jacquelyn J. Maher

My G. Mahoney

Ekaterina Maidji

Amy S. Major

Goldis Malek

Fransiska Malfait

James W. Mandell

Joseph L. Mankowski

Flavio Mantelli

Yves L. Marcel

Marcus M. Mareel

Benjamin Margolis

Claudio Marinho

Udo R. Markert

John C. Marshall

T. John Martin

Marco Marzioni

Koichi Matsuo

Christian M. Matter

Joshua T. Mattila

Theodora Mauro

Alain Mauviel

Valdemar Maximo

Robin J. McAnulty

Keith McCrae

Noel G. McElvaney

John K. McGuire

Marc D. McKee

Paul McMenamin

Iain Mcneish

Steve Meaney

Edgar Meinl

Marvin L. Meistrich

Fanyin Meng

Balaraj Menon

Giovanni Meola

Dan Mercola

Giampaolo Merlini

Albee Messing

Catherine Meyer-Schwesinger

David K. Meyerholz

George K. Michalopoulos

Bryan Michalowicz

Daniel Michele

Edoardo Midena

Ryan Miller

Jason C. Mills

David Milstone

John D. Minna

Anna Mitchell

Edward S. Mocarski, Jr.

Marcus J. Moeller

Makio Mogi

Roland Moll

Richard L. Momparler

Thomas J. Montine

Kathleen Montone

Dana Mordue

Carlos S. Moreno

Gilbert F. Morris

John S. Mort

Christopher A. Moskaluk

David Mu
Barbara M. Mueller

Mary J. Mulligan-Kehoe

Robert F. Mullins

Andrea Munsterberg

Walter L. Murfee

Mark W. Musch

James M. Musser

Fackson Mwale

Fnu Nagajyothi

Kanneboyina Nagaraju

Akinori Nakamura

Masahiko Nakamura

Toru Nakazawa

Masaomi Nangaku

Michal Neeman

Anne Negre-Salvayre

Andrew S. Neish

Kari Nejak-Bowen

Brad Nelson

Erik Nelson

William G. Nelson

Yin-Shan E. Ng

Jerry Y. Niederkorn

Alexander Y. Nikitin

David J. Nikolic-Paterson

Masato Nose

Warren B. Nothnick

Asma Nusrat

Patrick O'Connor

Timothy J. O'Leary

W. Charles O'Neill

Thomas G. Ohm

Hayato Ohshima

Joseph O. Ojo

Ghislain Opdenakker

Wayne Orr

Michael J. Overman

Gary K. Owens

Nades Palaniyar

Reinald Pamplona

Gyorgy Panyi

Ulf Panzer

Nazareno Paolocci

Joanne Paquin

Joo-Cheoil Park

William C. Parks

Tristram G. Parslow

Terence A. Partridge

Girish Patel

Rakesh Patel

Antoni Paul

Soumen Paul

Richard M. Peek, Jr.

Miguel A. Peinado

Harris Perlman

Guey-Chuen Perng

Mauro Perretti

Mercio A. Perrin

Jacques-Olivier Pers

Yuri Persidsky

Bryon E. Petersen

Janos Peti-Peterdi

Stephen C. Pflugfelder

Frank Pfrieger

Sem Phan

Michael P. Philpott

Maria M. Picken
Sonsoles Piera-Velazquez

Torsten Pietsch

Miguel A. Piris

Lucia Pirisi-Creek

Edyta C. Pirog

Costantino Pitzalis

Anna Plaas

Scott Plafker

Jeffrey L. Platt

Derek Pociask

Marc Poirot

Jurgen Pollheimer

Weston Porter

Miriam Postan

Lynne-Marie Postovit

Charalabos Pothoulakis

Sathyamangla V. Prasad

Richard J. Price

Richard W. Price

Alice Prince

Aaron Proweller

Devina Purmessur

Robert L. Raffai

Sasanka Ramanadham

Ramani Ramchandran

Ganesan Ramesh

Basabi Rana

Maria Pia Rastaldi

Ranjit Ray

Raju C. Reddy

Thomas Reiberger

Peter S. Reinach

Mark D. Rekhter

Alan T. Remaley

Daniel G. Remick

Giuseppe Remuzzi

Richard Rest

Sandra E. Reznik

Andrew Rice

Isabelle Richard

David W.H. Riches

Bruce L. Riser

Laure Rittié

Alain Rivard

Drucilla Roberts

Nicholas Roberts

Joseph A. Roche

Ulrich Rodeck

Jeffrey Rogers

Paola Romagnani

Roberto J. Romero

Martin J. Ronis

Noel R. Rose

Avi Rosenberg

Helene F. Rosenberg

Abdolmohamad Rostami

Chad J. Roy

Monika Roychowdhury

Lewis J. Rubin

M. Alanna Ruddell

Nancy H. Ruddle

Lawrence L. Rudel

David Rudnick

Bo R. Rueda

Urs T. Ruegg

Bernhard Ryffel

Miklos Sahin-Toth 
Magali Saint-Geniez

Taiji Sakamoto

David S. Salomon

Petri Salven

Francisco Sanchez-Madrid

Arun J. Sanyal

Debalina Sarkar

Abhay Satoskar

Lawrence J. Saubermann

Kenjiro Sawada

Stefano Schiaffino

Eberhard Schlatter

Laura S. Schmidt

Thomas Schmitz

William Schnaper

Gregory S. Schultz

Robert F. Schwabe

Timothy Seabrook

Rosalie Sears

Richard E. Seftor

Ekihiro Seki

Chandan K. Sen

John J. Shacka

Vijay Shah

Leslie C. Sharkey

Surendra Sharma

David Sheikh-Hamad

Chanjuan Shi

Zonggao Shi

Ie-Ming Shih

David T. Shima

Mari L. Shinohara

Yehuda Shoenfeld

Noah Shroyer

Nicholas E. Sibinga

Aleem Siddiqui

Roy L. Silverstein

Jean-Sébastien Silvestre

Fraser J. Sim

Ken J. Simpson

Rakesh K. Singh

Pravin C. Singhal

Alphonse E. Sirica

Stephen D. Skaper

Mihaela Skobe

Justine Smith

Matija Snuderl
Raymond A. Sobel

Keith R. Solomon

Pierre Sonveaux

Federica Sotgia

Andrew P. South

James R. Sowers

Janet R. Sparrow

Markus Sperandio

Carlo Spirli

Shanthi Srinivasan

Shiv Srivastava

Miguel Stadecker

Andrew W. Stadnyk

Rolf A. Stahl

William Stallcup

Ivan Stamenkovic

Ben Z. Stanger

Lucy Stead

Douglas A. Steeber

William G. Stetler-Stevenson

Christopher Stipp

Donna B. Stolz

James R. Stone

Adam C. Straub

Volker Straub

Dudley K. Strickland

Gary E. Striker

Olaf Stuve

Stephen P. Sugrue

David A. Sullivan

Holger Sultmann

Shoichiro Sumi

Tung-Tien Sun

John P. Sundberg

Gyongyi Szabo

Idikó Szabó

Clifford Taggart

Shin'ichi Takeda

James E. Talmadge

Veena Taneja

A. Keith Tanswell

Andrew W. Taylor

Cormac Taylor

Mauro M. Teixeira

Gregory H. Tesch

Neil Theise

Frank Thevenod
Henry J. Thompson

James G. Tidball

Peter G. Tipping

Jens Titze

David Tobin

Susanna Tomasoni

Marjana Tomic-Canic

Ian Tomlinson

Louis Tong

Warren G. Tourtellotte

Glenda Trujillo

Hidekazu Tsukamoto

Allan Tsung

Budd A. Tucker

Alda Tufro

Yasuo Uchiyama

Anna Valujskikh

Johan H.J.M. van Krieken

William E. Van Nostrand

Claudia R. van Roeyen

Richard S. Vander Heide

Radovan Vasko

Diego Vergani

Patrick Verrando

Joanne L. Viney

Harry V. Vinters

Renu Virmani

Gary A. Visner

Kristine S. Vogel

Angelika Vollmar

Katy Wack

David H. Walker

John L. Wallace

David Wallach

Gang Wang

Ke Wang

Qingde Wang

Xin W. Wang

David Warburton

Nicole L. Ward

Peter A. Ward

Gordon Warren

Mariusz A. Wasik

Christopher Weber

Steven A. Weinman

Louis M. Weiss

Alan Wells
Stephen E. Welty

Sabine Werner

James West

Judith A. West-Mays

Christof Westenfelder

Elizabeth M. Whitley

Clayton A. Wiley

Thomas M. Wilkie

Jennifer L. Wilkinson-Berka

Ifor Williams

Kenneth C. Williams

Dennis W. Wilson

Jeremy S. Wilson

Janusz Witowski

Hui Wu

T.C. $\mathrm{Wu}$

Xin Wu

Weiyun Wu

Peng Wu

Sandhya Xavier

Zhou Xing

Tesshi Yamada

Yutao Yan

Ivana V. Yang

Yi Yang

Cecelia C. Yates

Joanne E. Yi

Liya Yin

Stephen Yip

William H. Yong

Min You

Marian F. Young

Fu-Shin X. Yu

Yanlin Yu

C. Chris Yun

Stuart H. Yuspa

David Zagzag

Tamas Zakar

Ana C. Zenclussen

Frank Zhang

Jianhua Zhang

Haikuo Zhang

Hong-Bo Zhao

Jing Zheng

James D. Zieske

Berislav V. Zlokovic

Margot Zoller 